\title{
¿PUEDE HABLARSE DE CONSENSO CONSTRUCTIVISTA EN LA EDUCACIÓN CIENTÍFICA?
}

GIL PÉREZ, DANIEL ${ }^{1}$, CARRASCOSA ALÍS, JAIME ${ }^{1}$, DUMAS-CARRÉ, ANDRÉÉ2, FURIÓ MAS, CARLES ${ }^{1}$, GALLEGO, RÓMULO ${ }^{3}$, GENÉ DUCH, ANNA ${ }^{4}$, GONZÁLEZ, EDUARDO ${ }^{5}$, GUISASOLA, JENARO ${ }^{6}$, MARTÍNEZ-TORREGROSA, JOAQUÍN ${ }^{7}$, PESSOA DE CARVALHO, ANNA M. ${ }^{8}$, SALINAS, JULIA ${ }^{9}$, TRICÁRICO, HUGO ${ }^{10}$, y VALDÉS, PABLO ${ }^{11}$

${ }^{1}$ Universitat de València. España.

${ }^{2}$ IUFM d'Aix Marseille. Francia.

${ }^{3}$ Universidad Pedagógica Nacional. Colombia.

${ }^{4}$ Universitat de Lleida. España.

${ }^{5}$ Universitat de Córdoba. Argentina.

${ }^{6}$ Universidad del País Vasco. España.

${ }^{7}$ Universitat de Alicante. España.

${ }^{8}$ Universidade de São Paulo. Brasil.

${ }^{9}$ Univerdad de Tucumán. Argentina.

${ }^{10}$ Universidad de San Martín. Argentina.

${ }^{11}$ Instituto Superior Pedagógico EJ Varona. La Habana. Cuba.

\section{SUMMARY}

In this paper we analyse some of the criticisms that are being made to the «constructivist paradigm» and their implications on the development of the didactic of the sciences as a theoretical body knowledge.

\section{INTRODUCCIÓN}

Durante la última década hemos asistido, en la didáctica de las ciencias, al establecimiento de lo que Novak (1988) denominó un consenso emergente en torno a planteamientos constructivistas, calificados como la aportación más relevante de las últimas décadas en este campo (Gruender y Tobin, 1991). Parecía comenzar a superarse así el estatus «preparadigmático» que, todavía a comienzos de los ochenta, se atribuía a la didáctica de las ciencias (Klopfer, 1983). Como ha afirmado Hodson
(1992): «Hoy ya es posible construir un cuerpo de conocimientos en el que se integren coherentemente los distintos aspectos relativos a la enseñanza-aprendizaje de las ciencias.»

Todo parecía apuntar, pues, a la constitución de la didáctica de las ciencias (o, en terminología anglosajona, science education) como un nuevo campo de conocimiento (Aliberas, Gutiérrez e Izquierdo, 1989; 
Gil, 1993b y 1996; Martínez Terrades, 1998; Porlán, 1998; Gallego y Pérez Miranda, 1999). De hecho, el desarrollo de la didáctica de las ciencias a lo largo de las dos últimas décadas ha sido calificado de auténtica revolución (White, 1999). Se trata, por supuesto, de un desarrollo que, como en cualquier otro campo científico, no ha tenido un carácter lineal y en el que se han producido y siguen produciéndose fecundas controversias y reorientaciones más o menos profundas, pero que ha mostrado convergencias y progresos reales en la orientación del proceso de enseñanza-aprendizaje de las ciencias, refrendados por un notable volumen de investigaciones e innovaciones que pueden consultarse en las numerosas revistas especializadas hoy existentes y que han hecho posible ya la aparición de dos handbooks (Gabel, 1994; Fraser y Tobin, 1998).

Recientemente, sin embargo, comienzan a surgir voces que cuestionan los planteamientos constructivistas y la idea misma de avance hacia un cuerpo coherente de conocimientos en el campo de la didáctica de las ciencias (Suchting, 1992; Matthews, 1994; Solomon, 1994; Hardy y Taylor, 1997; Marín, 1999...).

Podría pensarse, pues, que el «consenso constructivista» no habría pasado de ser una nueva moda, una nueva «receta» fallida que nos devolvería, una vez más, pese a su probada ineficacia, al inamovible modelo de enseñanza-aprendizaje de las ciencias por transmisión/ recepción de conocimientos ya elaborados.

El objeto de este trabajo es analizar algunas de las críticas que se están emitiendo y estudiar sus implicaciones en el desarrollo de la didáctica de las ciencias como cuerpo coherente de conocimientos.

\section{LASPROPUESTASCONSTRUCTIVISTASNO CONSTITUYEN UNA RECETA}

Nos referiremos, en primer lugar, a interpretaciones demasiado simplistas y estereotipadas de propuestas que son presentadas a veces, injustificadamente, como quintaesencia de las orientaciones constructivistas. Cómo señalan Carretero y Limón (1996), «dichas propuestas suelen apoyarse en la convicción, más bien estólida, de que la aplicación de fórmulas del tipo tomemos los conocimientos previos del alumno, planteémosle conflictos cognitivos y modifiquémoslos solucionará fácilmente muchos problemas educativos». En el mismo sentido, Duit (1996) señala que «para algunos educadores en ciencias, el constructivismo se ha convertido en la nueva ideología capaz de solucionar cualquier problema de la enseñanza y el aprendizaje de las ciencias». Pero añade a continuación: «Sin ninguna duda se ha convertido también en una muy valiosa orientación para la educación en ciencias, tanto para su enseñanza como para la investigación en ese campo.»

De hecho, la crítica a esas visiones simplistas no puede considerarse como un cuestionamiento de los planteamientos constructivistas, sino que cuenta con una abun- dante literatura en el campo de la didáctica de las ciencias y se ha traducido en una profundización de dichos planteamientos. No debemos olvidar, sin embargo, que estas estrategias que hoy nos parecen fórmulas simplistas no fueron presentadas por sus autores de una forma tan esquemática (Posner et al., 1992; Pozo, 1989). Suponían un notable avance sobre otras fórmulas mucho más simplistas como la que subyace en el modelo de transmisión/recepción («expliquemos claramente los conocimientos, y los alumnos aprenderán») o en las ingenuas propuestas de «aprendizaje por descubrimiento inductivo» a partir de la experimentación autónoma de los alumnos -que algunos asocian erróneamente a Piaget (Marín, 1999b), pese a que este autor rechazó con contundencia «el mito del origen sensorial de los conocimientos científicos» (Piaget, 1971)- que fueron criticadas justa y fundamentadamente por numerosos autores (Ausubel, 1978, Giordan, 1978).

Las primeras propuestas de cambio conceptual, al menos, tenían en cuenta aspectos básicos del aprendizaje, en el sentido de que «todo aprendizaje depende de conocimientos previos» o que «quienes aprenden construyen significados» $y$ «establecen relaciones» (Resnick, 1983).

La mayor efectividad de estas estrategias sobre la simple transmisión de conocimientos ya elaborados fue refrendada por numerosas investigaciones realizadas en diferentes campos de las ciencias (Jung, 1993; Wandersee, Mintzes y Novak, 1994). Curiosamente, algunos críticos (Marín, 1999) ignoran u olvidan esta abundante literatura y se refieren únicamente a quienes mencionan dificultades, llegando a apoyar sus críticas con referencias a autores como Hashweh (1986), Duschl y Gitomer (1991) o Martínez Torregrosa, Domènech y Verdú (1993), cuyos trabajos constituyen profundizaciones en dichas estrategias y en modo alguno rechazo de las mismas. Profundizaciones necesarias, entre otras razones, porque pronto se constató que ciertas concepciones alternativas eran resistentes a la instrucción, incluso cuando ésta se orientaba explícitamente a producir el «cambio conceptual» (Fredette y Lochhead, 1981; Engel y Driver, 1986; Shuell, 1987; Hewson y Thorley, 1989...). Dicho con otras palabras: se hacía evidente que los indudables progresos logrados con las estrategias de cambio conceptual resultaban todavía insuficientes (Oliva, 1999).

Se comenzó así a comprender la necesidad, entre otros, de tomar en consideración las formas de razonamiento de los alumnos, superando el reduccionismo conceptual (Gil y Carrascosa, 1985; Hashweh, 1986; Cleminson, 1990; Duschl y Gitomer, 1991; Salinas, Cudmani y Pesa, 1996; Viennot, 1996...) y enriqueciendo así las propuestas constructivistas. Podemos recordar a este respecto las críticas realizadas por nosotros a las propuestas más simplistas de «cambio conceptual» (Gil et al., 1991; Gil, 1993).

La secuencia que proponen las estrategias de enseñanza basadas en el cambio conceptual consiste, como se recordará, en sacar a la luz las ideas de los alumnos, 
favoreciendo su formulación y clarificación, para después crear conflictos que las pongan en cuestión e introducir a continuación las concepciones científicas, cuya mayor potencia explicativa va a hacer posible el cambio conceptual. Es cierto que dicha estrategia puede, puntualmente, dar resultados positivos al llamar la atención sobre el peso de ciertas ideas de sentido común, asumidas acríticamente como evidencias; pero también es cierto que, practicada de forma reiterada, produce una inhibición y un rechazo muy comprensibles. En efecto, ¿qué sentido tiene hacer que los alumnos, una y otra vez, expliciten y afiancen sus ideas para seguidamente cuestionarlas? (Rela y Tricárico, 1997). ¿Cómo no ver en ello un artificio que aleja la situación de lo que constituye la construcción de conocimientos científicos? Esa construcción nunca se plantea para cuestionar ideas, para provocar cambios conceptuales, sino para resolver problemas de interés para los investigadores (es decir, en nuestro caso para los estudiantes); problemas que se abordan, como es lógico, a partir de los conocimientos que se poseen y de nuevas ideas que se construyen a título tentativo. En ese proceso, las concepciones iniciales podrán experimentar cambios e incluso, aunque más raramente, ser cuestionadas radicalmente -en su uso científico, bien entendido, sin que ello afecte necesariamente a su uso en el nivel cotidiano (Mortimer, 1995)-; pero ése no será nunca el objetivo, sino, repetimos, la resolución de los problemas planteados.

Desde un punto de vista constructivista resulta esencial asociar explícitamente la construcción de conocimientos a problemas $-\ll$ Todo conocimiento es la respuesta a una pregunta.» (Bachelard, 1938)- y ello cuestiona de forma radical estas estrategias de cambio conceptual en lo que supone tomar las ideas de los alumnos como punto de partida y concebir el cambio conceptual como $\gg$ mero desplazamiento de ideas» (Oliva, 1999). Por otra parte, una característica fundamental del tratamiento científico de los problemas es tomar las ideas que se tienen -incluso las más seguras y obvias- como simples hipótesis de trabajo que es necesario controlar, esforzándose en imaginar otras hipótesis, etc. Ello concede un estatus muy diferente a las situaciones de conflicto cognoscitivo: ya no suponen para los estudiantes el cuestionamiento externo de las ideas personales, ni la reiterada aceptación de las insuficiencias del propio pensamiento (con las consiguientes implicaciones afectivas), sino un trabajo de profundización en el que unas ideas (tomadas como hipótesis) son sustituidas por otras (tan personales como las anteriores).

No se trata, como puede verse, de eliminar los conflictos cognoscitivos, sino de evitar que adquieran el carácter de una confrontación entre las ideas propias (incorrectas) y los conocimientos científicos (externos). A este respecto, Solomon (1991) argumenta que «tras impulsar la expresión de un conjunto de opiniones particulares, el profesor no puede simplemente rechazar las que no se ajustan a la teoría vigente. De ese modo dejaría de ser posible un diálogo abierto».

Por todo ello, la estrategia de enseñanza que nos parece más coherente con la orientación constructivista y con las características del razonamiento científico es la que plantea el aprendizaje como tratamiento de situaciones problemáticas abiertas que los alumnos puedan considerar de interés (Furió y Gil, 1978; Driver y Oldham, 1986; Gil y Martínez-Torregrosa, 1987; Burbules y Linn, 1991; Gené, 1991; Gil et al., 1991; Duschl, 1990 y 1995; Wheatley, 1991; Pessoa de Carvalho y Gil, 1995; National Research Council, 1996; Guisasola y de la Iglesia, 1997; Furió y Guisasola, 1998; Jiménez, 1998 ...). Un tratamiento con aspiración científica no puede traducirse en fórmulas sencillas, sino que ha de contemplarse, insistimos, como una actividad científica, abierta $y$ creativa, debidamente orientada por el profesor (Hodson, 1992), que incluya, entre otros:

- La consideración del posible interés y relevancia de las situaciones propuestas que dé sentido a su estudio y evite que los alumnos se vean sumergidos en el tratamiento de una situación sin haber podido siquiera formarse una primera idea motivadora.

- El estudio cualitativo de las situaciones problemáticas planteadas y la toma de decisiones, para acotar problemas y operativizar qué es lo que se busca (ocasión para que los estudiantes comiencen a explicitar funcionalmente sus concepciones).

- La invención de conceptos y emisión de hipótesis, (ocasión para que las ideas previas sean utilizadas para hacer predicciones susceptibles de ser sometidas a prueba).

- La elaboración de estrategias de resolución (incluyendo, en su caso, diseños experimentales) para someter a prueba las hipótesis, a la luz del cuerpo de conocimientos de que se dispone.

- La resolución y el análisis de los resultados, cotejándolos con los obtenidos por otros grupos de estudiantes y por la comunidad científica. Ello puede convertirse en ocasión de conflicto cognoscitivo entre distintas concepciones (tomadas todas ellas como hipótesis) y obligar a concebir nuevas conjeturas y a replantear la investigación.

- El manejo reiterado de los nuevos conocimientos en una variedad de situaciones, poniendo un énfasis especial en las relaciones ciencia-técnología-sociedad que enmarcan el desarrollo científico (propiciando, a este respecto, la toma de decisiones) y dirigiendo todo este tratamiento a mostrar el carácter de cuerpo coherente que tiene toda ciencia, favoreciendo, para ello, las actividades de síntesis (esquemas, memorias, recapitulaciones, mapas conceptuales...), la elaboración de productos (susceptibles de romper con planteamientos excesivamente escolares y de reforzar el interés por la tarea) y la concepción de nuevos problemas.

Es conveniente remarcar que las orientaciones precedentes no constituyen un algoritmo que pretenda guiar paso a paso la actividad de los alumnos, sino indicaciones genéricas que llaman la atención sobre aspectos esenciales en la construcción de conocimientos científi- 
cos que, a menudo, no son suficientemente tenidos en cuenta en la enseñanza de las ciencias. Nos referimos tanto a los aspectos metodológicos como a los problemas de contextualización del trabajo científico (relaciones CTS, toma de decisiones...) y a los componentes afectivos (interés por la tarea, clima de trabajo...). El aprendizaje de las ciencias es concebido, así, no como un simple cambio conceptual, sino como un cambio a la vez conceptual, metodológico y axiológico (Gallego y Pérez Miranda, 1994) o, mejor, como un proceso de investigación orientada que permite a los alumnos participar en la «re-construcción» de los conocimientos científicos (que habitualmente la enseñanza transmite ya elaborados), lo cual favorece un aprendizaje más eficiente y significativo.

Como puede verse, los planteamientos constructivistas en la enseñanza de las ciencias están lejos de las recetas simplistas, justamente criticadas por Carretero y Limón (1996), que en modo alguno pueden presentarse como expresión de dichos planteamientos.

Otras críticas, sin embargo, se están dirigiendo hoy, al menos aparentemente, hacia los fundamentos mismos de las propuestas constructivistas. Son esas críticas las que queremos analizar aquí, centrándonos en artículos como los de Suchting, Matthews o Solomon, de títulos muy significativos: Constructivism deconstructed (Suchting, 1992), Vino viejo en botellas nuevas. Un problema con la epistemología constructivista (Matthews, 1994), The rise and fall of constructivism (Solomon, 1994)...

\section{¿DE QUÉ CONSTRUCTIVISMO ESTAMOS HABLANDO?}

En su artículo «El constructivismo deconstruido», Suchting (1992) comienza aclarando que «trata de una doctrina, el constructivismo, que durante algún tiempo ha ejercido una fuerte influencia en educación y de la que considera creador y principal exponente a Ernst von Glaserfeld».

Resulta curioso ver cómo Suchting habla del constructivismo en pasado, dando por sentado, sin justificación alguna, que su influencia se ejerció «durante algún tiempo». Pero lo esencial es señalar que todo el artículo está centrado en la crítica de las tesis filosóficas de von Glaserfeld, llegando a la conclusión de que «los conceptos y tesis fundamentales del constructivismo son muy oscuros y están escasa e insatisfactoriamente fundamentados».

Sin entrar a discutir el posible interés de críticas como la de Suchting, hemos de señalar que ese debate en torno a las tesis de von Glaserfeld tiene poco que ver con las propuestas constructivistas en el campo de la enseñanza-aprendizaje de las ciencias. De hecho, el artículo de Suchting no contiene una sola cita procedente de este campo, que parece desconocer por completo, hasta el punto de considerar a von Glaserfeld, cuyo nombre ha comenzado a ser citado tan sólo recientemente, el «ori- ginator». Éste es un primer y grave defecto de algunas de las críticas actuales: «apuntan» hacia otro blanco e ignoran las contribuciones en el campo de la didáctica de las ciencias.

Podemos afirmar, pues, que el debate que plantean Suchting y otros autores (Nola, 1997; Hardy y Taylor, $1997 . .$.$) no es nuestro debate. Con ello no pretendemos$ negar el interés de estudiar los trabajos de von Glaserfeld y sus posibles aportaciones a las controversias en torno a las propuestas constructivistas en el campo de la enseñanza-aprendizaje de las ciencias. Pero no podemos aceptar una discusión en términos genéricos que dejen suponer, como hace Suchting, que hablamos de constructivismo «en general» y que estamos «aplicando» las tesis de von Glaserfeld.

La crítica de Solomon (1994) tiene, sin duda, otro carácter, puesto que procede de una voz autorizada en el campo de la didáctica de las ciencias. Solomon reconoce que los planteamientos constructivistas en didáctica de las ciencias tienen su origen en las investigaciones realizadas en torno a los problemas de enseñanza-aprendizaje de las ciencias. De hecho, Solomon asocia el surgimiento de esta corriente a la publicación del artículo de Driver y Easley (1978) «Pupils \& paradigms: a review of literature related to concept development in adolescent science students». Pero, a continuación, Solomon señala que, a principios de los años ochenta, «se descubrió que lo que podemos denominar el cuerpo teórico había sido escrito cerca de treinta años antes por George Kelly».

Fijémonos que Solomon no dice que los trabajos de Kelly apoyaban las nuevas ideas, sino que constituían su cuerpo teórico. Se trata, a nuestro entender, de un grave error que niega la posibilidad de que la investigación en didáctica de las ciencias dé lugar a un cuerpo específico de conocimientos y reduce su fundamentación a la aplicación de conocimientos externos. Conviene aclarar que, al afirmar la existencia de la didáctica de las ciencias como cuerpo específico de conocimientos, no pretendemos ignorar las contribuciones de otros dominios como la psicología educativa o la historia de las ciencias. Muy al contrario, es la existencia de un cuerpo específico de conocimientos lo que hace posible la integración de esas contribuciones, sin caer en aplicaciones mecánicas escasamente efectivas (Gil, 1993b).

En nuestra opinión, algunas ideas de Kelly pueden resultar sugerentes y ayudar a la construcción del nuevo cuerpo de conocimientos en torno a los problemas de enseñanza-aprendizaje de las ciencias, pero sus reflexiones no estaban centradas en el campo de la enseñanza de la ciencia y no tiene sentido plantear su mera aplicación en dicho campo.

Sin embargo, las críticas de Solomon a los planteamientos constructivistas se centran en las contribuciones de Kelly y otros autores como Glasersfeld, igualmente externos al campo de la didáctica de las ciencias. Muy en particular, Solomon se centra en mostrar las limitaciones de la metáfora de Kelly «every man his own scientist», 
dando por supuesto que el constructivismo «se basaba, en esencia, en la noción del estudiante como científico». Solomon admite, además, como lógico corolario, que ello supone dejar de lado la adquisición de cuerpos de conocimientos.

Pero la idea del estudiante como científico es una metáfora cuyas limitaciones han sido señaladas también desde el campo de la didáctica de las ciencias y, más específicamente, desde los planteamientos constructivistas, porque no expresa adecuadamente lo que la investigación ha mostrado acerca del proceso de enseñanza-aprendizaje de las ciencias: es difícil no estar de acuerdo en que los alumnos por sí solos no pueden construir todos los conocimientos científicos. Como señala Pozo (1987), «es bien cierto que muchos de los conceptos centrales de la ciencia son bastantes difíciles de descubrir para la mayor parte si no para la totalidad de los adolescentes e incluso de los adultos universitarios». Sin embargo, como hemos argumentado en diversos trabajos (Gil et al., 1991; Gil, 1993 y 1997), de aquí no se sigue que se haya de recurrir necesariamente a la transmisión de dichos conocimientos ni que se haya de poner en cuestión las orientaciones constructivistas. En efecto, es bien sabido que, cuando alguien se incorpora a un equipo de investigadores, puede alcanzar con relativa rapidez el nivel medio del resto del equipo. Y ello no mediante una transmisión verbal, sino abordando problemas en los que quienes actúan de directores/ formadores son expertos. La situación cambia, por supuesto, cuando se abordan problemas que son nuevos para todos. El avance, si lo hay, se hace entonces lento y sinuoso. La propuesta de organizar el aprendizaje de los alumnos como una construcción de conocimientos responde a la primera de las situaciones, es decir, a la de una investigación orientada, en dominios perfectamente conocidos por el «director de investigaciones» (profesor) y en la que los resultados parciales, embrionarios, obtenidos por los alumnos, pueden ser reforzados, matizados o puestos en cuestión por los obtenidos por los científicos que les han precedido. No se trata, pues, de «engañar» a los alumnos, de hacerles creer que los conocimientos se construyen con la aparente facilidad con que ellos los adquieren (Hodson, 1985), sino de colocarles en una situación por la que los científicos habitualmente pasan durante su formación, y durante la que podrán familiarizarse mínimamente con lo que es el trabajo científico y sus resultados, replicando para ello investigaciones ya realizadas por otros, abordando, en definitiva, problemas conocidos por quienes dirigen su trabajo. Como afirma Hodson (1992): «Los estudiantes desarrollan mejor su comprensión conceptual y aprenden más acerca de la naturaleza de la ciencia cuando participan en investigaciones científicas, con tal de que haya suficientes oportunidades y apoyo para la investigación.»

El planteamiento constructivista del aprendizaje de las ciencias ha de responder, pensamos, a estas características de investigación orientada: un trabajo de investigación en el que constantemente se cotejan los resultados de los distintos equipos y se cuenta con la inestimable ayuda de un experto. No creemos necesario insistir aquí en los bien conocidos y documentados argumentos en favor del trabajo en pequeños grupos como forma de incrementar el nivel de participación y la creatividad necesaria para abordar situaciones no familiares y abiertas (Ausubel, 1978; Solomon, 1987; Linn, 1987; Burbules y Linn, 1991; Robinson y Niaz, 1991...), como indudablemente son las concebidas para posibilitar la construcción de conocimientos. Sí queremos insistir, por el contrario, en la necesidad, mucho menos tenida en cuenta, de favorecer la máxima interacción entre los equipos (Gil et al., 1991; Wheatley, 1991; Kempa y Ayob, 1995), a través de la cual los alumnos pueden asomarse a una característica fundamental del trabajo científico: la insuficiencia de las ideas y resultados obtenidos por un único colectivo y la necesidad de cotejarlos con los obtenidos por otros, hasta que se produzca suficiente evidencia convergente para que la comunidad científica los acepte. Nunca se insistirá bastante, en efecto, en que, por ejemplo, unos pocos resultados experimentales como los que se pueden obtener en un laboratorio escolar no permiten hablar de verificación de hipótesis (Hodson, 1985); de ahí la importancia de los intercambios intergrupos y la participación del profesor como «portavoz de otros muchos investigadores», es decir, de lo que la comunidad científica ha ido aceptando como resultado de un largo y difícil proceso. En definitiva, «las propuestas constructivistas en el campo de la educación científica se concretan en un trabajo colectivo de investigación orientada, tan alejado del descubrimiento autónomo como de la transmisión de conocimientos ya elaborados» (Gil, 1983; Millar y Driver, 1987). Dicho con otras palabras: entre la metáfora del alumno como simple receptor y la que lo asimila, siguiendo a Kelly, a un «investigador» autónomo (Pope y Gilbert, 1983; Solomon, 1994), proponemos la metáfora del «investigador novel» que integra coherentemente, además, las aportaciones de Vigotskii sobre la «zona de desarrollo potencial» y el papel del adulto en el aprendizaje (Howe, 1996). Las situaciones problemáticas abiertas, el trabajo científico en equipo y la interacción entre los equipos se convierten así en tres elementos esenciales de una orientación del aprendizaje de las ciencias que llegamos a denominar constructivista radical (Gil, 1993; Furió, 1994).

Nos apresuramos a aclarar que no hablábamos de constructivismo radical en el sentido que da von Glaserfeld a dicha expresión (rechazo del realismo ontológico, es decir, rechazo de la idea de que los constructos son una réplica o reflejo de estructuras que existen independientemente de nuestro pensamiento). Lo que nosotros denominábamos una orientación radicalmente constructivista es una propuesta que contempla una participación activa de los estudiantes en la construcción de los conocimientos y no la simple reconstrucción personal de los conocimientos proporcionados, ya elaborados, por el profesor o el texto. Ello conecta con lo que algunos escribíamos ya en 1978, cuando desconocíamos incluso la expresión constructivismo: «se trata, en la medida de lo posible, de colocar a los alumnos en situación de producir conocimientos, de explorar alternativas, superando la mera asimilación de conocimientos ya elaborados» (Furió y Gil, 1978). Aunque hablába- 
mos de «producir» y no de «construir» conocimientos, un párrafo como el anterior está mucho más cerca de las actuales propuestas constructivistas en la enseñanza de las ciencias que las reflexiones de Kelly o de von Glaserfeld (pese a su coincidencia en el uso de expresiones como construcción de conocimientos). Y lo mismo cabe decir de, por ejemplo, generative learning model (Osborne y Wittrok, 1985), que, aunque utiliza una terminología distinta a la habitual, constituye una propuesta coherente con lo que entendemos por construcción de conocimientos en la educación científica. Como ha señalado Duit (1996), «hay una tradición constructivista (bajo diferentes denominaciones, por supuesto) tanto en pedagogía como en didáctica de las ciencias que se remonta bastante lejos en la historia de la educación».

Consideramos, pues, que la argumentación de Solomon contra los planteamientos constructivistas en la enseñanza de las ciencias adolece de serias limitaciones, puesto que dirige sus ataques contra algo que no expresa los avances de la investigación en este campo e ignora numerosas contribuciones que sí se proponen la construcción de cuerpos de conocimientos, como, por ejemplo, las de Viennot (1989, 1996 y 1999), o McDermott y otros (1996) o las que numerosos equipos han realizado en el campo de las prácticas de laboratorio, de la resolución de problemas de lápiz y papel, de la evaluación, etc., que se integran coherentemente en una estrategia de aprendizaje de las ciencias como investigación orientada (Gil et al., 1999). Toda esta investigación -recogida en las revistas internacionales, libros colectivos (Tiberghien, Jossem y Barojas, 1998) y en los dos handbooks aparecidos (Gabel, 1994; Fraser y Tobin, 1998) - es la que permite hablar de consenso emergente, pero no es tenida en cuenta por Solomon, ni mucho menos por autores como Suchting (1992).

Sin embargo, el debate que artículos como éste generan resulta de gran interés, puesto que conecta con las reticencias de muchos profesores de ciencias a las propuestas constructivistas, interpretadas, incorrectamente, como la aceptación de la metáfora de «los estudiantes como científicos», a las que es preciso dar una respuesta fundamentada.

Artículos como el de Solomon muestran, además, el peligro de una fundamentación teórica de la didáctica de las ciencias que se reduzca a una simple adscripción a un cuerpo de conocimientos externos. Es necesario construir un cuerpo de conocimientos propio en torno a los problemas específicos de enseñanza-aprendizaje de las ciencias. Ésa es, pensamos, la orientación más fructífera, teniendo en cuenta que, como Carretero señalaba en el prólogo a El aprendizaje de la ciencia y pensamiento causal (Pozo, 1987), «no se puede hablar del pensamiento de los sujetos al margen del contenido de los problemas». La fundamentación del cuerpo de conocimientos, en construcción, que aborda la problemática de la educación científica habrá de ser, está siendo ya, una fundamentación específica, no una simple adscripción a otros campos de conocimiento. Ello no significa, insistimos una vez más, ignorar las aportaciones de esos otros dominios que pueden, por supuesto, inspirar, cuestionar, etc. el trabajo realizado en didáctica de las ciencias, pero que no pueden «aplicarse» sin más. Podemos mencionar, en ese mismo sentido, que también los avances de la neurociencia parecen apoyar también, según algunos autores (Hendry y King, 1994; Anderson, 1997; Roth, 1998), las orientaciones constructivistas, al tiempo que muestran las limitaciones de los modelos de transmisión y de procesado de la información.

Más aún, resulta razonable suponer que las aportaciones realizadas por la investigación en torno a los problemas que plantea el proceso de enseñanza-aprendizaje de las ciencias, aunque resulten específicas, no pueden resultar radicalmente contradictorias con los hallazgos de la psicología educativa, la epistemología genética, la neurociencia... o lo que muestra la historia y la filosofía de la ciencia acerca de cómo se construye el conocimiento científico. Por ello, a la pregunta con que Marín (1999) termina su reflexión sobre el cambio conceptual ( « ¿Por qué hacer una propuesta didáctica partiendo de una supuesta analogía entre desarrollo científico y cognición del alumno cuando es posible hacerla con datos y argumentos teóricos tomados directamente de éste?»), nosotros replicamos: ¿Es posible plantear correctamente el aprendizaje de las ciencias sin tomar en consideración la naturaleza de la construcción de los conocimientos científicos?

Nadie propone limitar la fundamentación de las estrategias de la enseñanza de las ciencias a las aportaciones de la historia y filosofía de la ciencia, puesto que «reconstruir» conocimientos científicos en el aula no es lo mismo, claro está, que investigar en la frontera del conocimiento (Porlán, 1993; Izquierdo, Sanmanrtín y Espinet, 1999). Pero, como ha mostrado una potente línea de investigación en torno a las concepciones docentes espontáneas sobre la ciencia (Bell y Pearson, 1992, Désauteles et al., 1993; Guilbert y Meloche, 1993...), la comprensión y toma en consideración de la actividad científica por los docentes aparece como conditio sine qua non -aunque no suficiente (Hodson, 1993)- para una enseñanza de las ciencias realmente eficiente.

Intentar ignorar la historia y la epistemología de la ciencia en la fundamentación de las estrategias de la enseñanza de las ciencias nos parece un grave reduccionismo, tanto más cuanto que, como recordábamos ya a principios de los años ochenta (Gil, 1983), las investigaciones realizadas por Piaget y sus colaboradores en el Instituto de Epistemología Genética (Piaget, 1970) han mostrado el paralelismo entre la evolución histórica de una ciencia y la adquisición de las ideas correspondientes en el niño (Wandersee, Mintzes y Novak, 1994). Así, por ejemplo, estos estudios han dejado patente cómo las experiencias cotidianas sobre el movimiento de los cuerpos generan una visión del comportamiento mecánico de la materia muy próxima a la física aristotélico-escolástica (Whitaker, 1983). En cualquier caso resulta paradójico, señala Oliva (1999b), que tomando Marín a Piaget como referente «se cuestione tajantemente el paralelismo entre ontogénesis y filogénesis, el cual resulta ciertamente uno de los puntos claves sobre los que se asienta la epistemología genética». La «recuperación»de 
Piaget, en la que estamos absolutamente de acuerdo, exige no ignorar aportaciones tan fundamentales como ésta o su crítica al origen sensorial del conocimiento y, por tanto, a las propuestas de aprendizaje «por descubrimiento inductivo y autónomo».

\section{CONCLUSIÓN}

Queremos llamar la atención, para terminar, sobre el peligro de las lecturas superficiales que adscriben la orientación constructivista en la educación científica a las ideas de determinados pensadores, que olvidan el importante trabajo de integración y síntesis que se está desarrollando en el área de la educación científica y que llevan la discusión fuera de la problemática concreta de la enseñanza-aprendizaje de las ciencias. De esa forma, como Carretaro y Limón (1996) han señalado, «la amplia aplicación del término constructivismo en diferentes contextos parece haberlo dotado de una cierta generalidad y vaguedad» (Pozo et al., 1991; Sanmartí y Azcárate, 1997). Una vaguedad que, añadamos, permite, incluso, calificar como «constructivista» lo que cada cual ha hecho siempre («yo explico los conocimientos y mis alumnos los reconstruyen en su cabeza»). Ésa es una lectura que tiene, sin duda, sus adeptos: el constructivismo no sería sino una interpretación del aprendizaje y no tendría nada que decir acerca de la enseñanza. (Dicho de otro modo: dejemos las cosas como están).

Quizás esta vaguedad (esta conjunción, bajo el paraguas constructivista, de recetas simplistas, de discusiones filosóficas alejadas de la problemática concreta de la enseñanza-aprendizaje de las ciencias, de interpretaciones light que permiten a cualquiera, haga lo que haga, denominarse constructivista...) es lo que impulsa a autores como Osborne (1996) a hablar de beyond constructivism y a Giordan (1996) a preguntarse «¿cómo ir más allá de los modelos constructivistas?» y a proponer nuevas denominaciones (modelo alostérico).

En nuestra opinión, sin embargo, la expresión de un consenso constructivista (Resnick, 1983; Novak, 1988) sigue siendo útil para poner de relieve la convergencia

\section{REFERENCIAS BIBLIOGRÁFICAS}

ALIBERAS, J., GUTIÉRREZ, R. e IZQUIERDO, M. (1989). La didàctica de les ciències: una empresa racional. Enseñanza de las Ciencias, 7(3), pp. 277-284.

ANDERSON, O.R. (1997). A neurocognitive perspective on current learning theory and science instructional strategies. Science Education, 81, pp. 67-89.

AUSUBEL D.P. (1978). Psicología educativa. Un punto de vista cognoscitivo. México: Trillas. básica, en el campo de la didáctica de las ciencias, de propuestas, tan diversas terminológicamente, como las de Posner y otros (1982), Osborne y Wittrock (1983), Driver y Oldham (1986), Giordan (1989), Duschl y Gitomer (1991), Hodson (1992)... Una convergencia que apoya la idea de avance-no exento de controversias, como en cualquier campo científico- hacia la construcción de un nuevo modelo de enseñanza-aprendizaje de las ciencias capaz de desplazar al de simple transmisión/ recepción de conocimientos ya elaborados y, en definitiva, de avance hacia la conformación de la didáctica de las ciencias como un nuevo campo de conocimiento (Martínez Terrades, 1998; White, 1999). Es preciso añadir que ello no será una tarea simple. Como ha señalado Duit (1996), la investigación ha mostrado con claridad que los profesores ofrecen serias resistencias a adoptar los planteamientos constructivistas e introducen, a menudo, graves distorsiones. ¿Qué sentido tiene, por ejemplo, hablar de «aprendizaje como investigación orientada» si los profesores carecen de experiencia investigadora? (Dumas Carré y Weil Barais, 1998). Ello nos remite al problema de la formación del profesorado y a la necesidad de implicarle en la «re-construcción» de las propuestas didácticas (Gil, Furió y Gavidia, 1998).

No quisiéramos terminar estas reflexiones sin expresar nuestro convencimiento de que la didáctica de las ciencias está aún en sus primeros balbuceos teóricos. Quizás debates como éste hagan sonreír dentro de algunos años, pero son expresión de la pasión con la que muchos de nosotros nos planteamos la necesidad de cambiar la enseñanza de las ciencias para lograr aprendizajes más significativos y actitudes más positivas. Mucho más importante que las discrepancias es la voluntad de seguir profundizando, de no contentarse con los pobres resultados que han convertido la enseñanza de las ciencias en un problema social. Sigamos, pues, investigando e innovando, sin perder de vista lo que está en juego: la formación científica de los futuros ciudadanos y ciudadanas para hacer posible su participación en la toma fundamentada de decisiones. El que existan enfoques diferentes e incluso radicalmente enfrentados es, en definitiva, positivo... si se traduce en autoexigencia para seguir profundizando y cotejar nuestros desarrollos parciales con los globales de una comunidad científica cada vez más amplia y rigurosa.
BACHELARD, G. (1938). Laformation de l'espritscientifique. París: Vrin.

BELL, B.F. y PEARSON, J. (1992). Better Learning. International Journal of Science Education, 14(3), pp. 349-361

BURBULES, N. y LINN, M. (1991). Science education and philosophy of science: congruence or contradiction? International Journal of Science Education, 13(3), pp. 227-241. 
CARRETERO, M. y LIMÓN, M. (1996). Problemas actuales del constructivismo. De la teoría a la práctica, en Rodrigo M.J. y Arnay (eds.). La construcción del conocimiento escolar. Ecos de un debate. Buenos Aires: Auque.

CLEMINSON, A. (1991). Establishing an epistemological base for science teaching in the light of contemporary notions of the nature of science and of how children learn science. Journal of Research in Science Teaching, 27(5), pp. 429- 445.

DÉSAUTELS, J., LAROCHELLE, M., GAGNÉ, B. y RUEL, F. (1993). La formation à l'enseignement des sciences: le virage épistémologique. Didaskalia, 1, pp. 49-67

DRIVER, R. y EASLEY, J. (1978). Pupils and paradigms: A review of literature related to concept development in adolescent science students. Studies in Science Education, 10, pp. 37-70.

DRIVER, R. y OLDHAM, V. (1986). A constructivist approach to curriculum development in science. Studies in Science Education, 13, pp. 105-122.

DUIT, R. (1996). The constructivist view in science education. What it has to offer and what should not be expected from it. Investigaçôes em ensino de ciências, 1, pp. 40-75.

DUMAS-CARRÉ, A. y WEIL-BARAIS, A. (1998). Tutelle et médiation dans l'education scientifique. Berna: Peter Lang.

DUSCHL, R. (1990). Restructuring science education: The role of theories and their importance. Nueva York: Teacher College Press, Columbia University.

DUSCHL, R. (1995). Más allá del conocimiento: los desafíos epistemológicos y sociales de la enseñanza mediante el cambio conceptual. Enseñanza de las Ciencias, 13(1), pp. 3-14.

DUSCHL, R. y GITOMER, D. (1991). Epistemological Perspectives on conceptual change: implications for educational practice. Journal of Research in Science Teaching, 28(9), pp. 839-858.

ENGEL, E. y DRIVER, R. (1986). A study of consistency in the use of students' conceptual framework across different task contexts. Science Education, 70(4), pp. 473-496.

ERNST, P. (1993). Constructivism, the psychology of learning and the nature of mathematics: some critical issues. Science \& Education, 2, pp. 87-93.

FRASER, B. y TOBIN, K.G. (eds.). (1998). International Handbook of Science Education. Londres: Kluber Academic Publishers.

FREDETTE, N. y LOCHHEAD, J. (1981). Students conceptions of electric current. The Physics Teacher, 18, pp. 194-198.

FURIÓ, C. (1994). La enseñanza de las ciencias como investigación: un modelo emergente, en Proceedings of the International Conference «Science andMathematics Education for the $21^{\text {st }}$ Century: towards innovatory approaches», I, pp. 158-159.

FURIÓ, C. y GIL, D. (1978). El programa-guía: una propuesta para la renovación de la didáctica de la física y química. ICE de la Universidad de Valencia.

FURIÓ, C. y GUISASOLA, J. (1998). Construcción del concepto de potencial eléctrico mediante el aprendizaje por investigación. Revista de Enseñanza de la Física, 11(1), pp. 25-37.

GABEL, D.L. (ed.) (1994). Handbook of Research on Science Teaching and Learning. Nueva York: MacMillan Pub Co.
GALLEGO, R. y PÉREZ MIRANDA, R. (1994). Representaciones y conceptos científicos: Un programade investigación Bogotá: Universidad Pedagógica Nacional.

GALLEGO, R. y PÉREZ MIRANDA, R. (1999).Aprendibilidad, enseñabilidad y educabilidad. Una discusión. Bogotá: Universidad Pedagógica Nacional.

GENÉ, A. (1991). Cambio conceptual y metodológico en la enseñanza y el aprendizaje de la evolución de los seres vivos. Un ejemplo concreto. Enseñanza de las Ciencias, 9(1), pp. 22-27.

GIL, D. (1983). Tres paradigmas básicos en la enseñanza de las ciencias. Enseñanza de las Ciencias, 1(1), pp. 26-33.

GIL, D. (1993). Contribución de la historia y filosofía de las ciencias al desarrollo de un modelo de enseñanza-aprendizaje como investigación. Enseñanza de las Ciencias, 11(2), pp. 197-212

GIL, D. (1993b). Psicología educativa y didáctica de las ciencias: los procesos de enseñanza-aprendizaje de las ciencias como lugar de encuentro. Infancia y Aprendizaje, pp. 62-63.

GIL, D. (1996). New trends in science education. International Journal of Science Education, 18, pp. 889-901.

GIL, D. (1997). ¿Crisis en los planteamientos constructivistas?, en Ospina, H.F. y López, L. (eds.). Pedagogías constructivistas, pedagogías activas y desarrollo humano. Bogotá: Cooperativa Editorial Magisterio.

GIL, D. y CARRASCOSA, J. (1985). Science learning as a conceptual and methodological change. European Journal of Science Education, 7(3), pp. 231-236.

GIL, D., CARRASCOSA, J., FURIÓ, C. y MARTÍNEZTORREGROSA, J. (1991). La enseñanza de las ciencias en la educación secundaria. Barcelona: Horsori.

GIL, D., FURIÓ, C., VALDÉS, P., SALINAS, J., MARTÍNEZTORREGROSA, J., GUISASOLA, J., GONZÁLEZ, E. DUMAS-CARRE, A., GOFFARD, M. y PESSOA DE CARVALHO, A. (1999). ¿Tiene sentido seguir distinguiendo entre aprendizaje de conceptos, resolución de problemas de lápiz y papel y realización de prácticas de laboratorio? Enseñanza de las Ciencias, 17(2).

GIL, D. y MARTÍNEZ-TORREGROSA, J. (1987). Los programas-guía de actividades: una concreción del modelo constructivista de aprendizaje de las ciencias. Investigación en la Escuela, 3, pp. 3-12

GIORDAN, A. (1978). Observation-Experimentation: mais comment les élèves apprennet-ils? Revue Francaise de Pedagogie, 44, pp. 66-73. Traducción cast. (1978) en Infancia y Aprendizaje, 13.

GIORDAN, A. (1989). De las concepciones de los alumnos a un modelo de aprendizaje alostérico. Investigación en la Escuela, 8, pp. 3-14.

GIORDAN, A. (1996). ¿Cómo ir más allá de los modelos constructivistas? La utilización didáctica de las concepciones de los estudiantes. Investigación en la Escuela, 28, pp. 7-22.

GRUENDER, C.D. y TOBIN, K. (1991). Promise and Prospect, Science Education, 75(1), pp. 1-8

GUILBERT, L. y MELOCHE, D. (1993). L'idée de science chez des enseignants en formation: un lien entre l'histoire des sciences et l'hétérogénéité des visions. Didaskalia, 2, pp. 7-30. 
GUISASOLA,J. y DELA IGLESIA, R. (1997). «Erein Projectua»: Proyecto de Ciencias para la ESO basado en el planteamiento de situaciones problemáticas. Alambique, 13, pp. 83-93.

HARDY, M.D. y TAYLOR, P.C. (1997). Von Glaserfeld's Radical Constructivism: A Critical Review. Science \& Education, 6, pp. 135-150.

HASHWEH, M.Z. (1986). Towards an explanation of conceptual change. European Journal of Science Education, 8(3), pp. 229-249.

HENDRY, G.D. y KING, R.C. (1994). On theory of learning and knowledge: educational implications of advances on neuroscience, in science education. Science Education, 8(3), pp. 223-253.

HEWSON, P.W. y THORLEY, N.R. (1989). The conditions of conceptual change. International Journal of Science Education, 11 , pp. 541-553.

HODSON, D. (1985). Philosophy of science, science and science education. Studies in Science Education, 12, pp. 25-57.

HODSON, D. (1988). Towards a philosophically more valid science curriculum. Science Education, 72(1), pp. 19-40.

HODSON, D. (1992). In search of a meaningful relationship: an exploration of some issues relating to integration in science and science education. International Journal of Science Education, 14(5), pp. 541-566.

HODSON, D. (1993). Philosophic stance of secondary school science teachers, curriculum experiences and children's understanding of science: some preliminary findings. Interchange, 24(1\&2), pp. 41-52.

HODSON, D. (1996). Practical work in school science: exploring some directions for change. International Journal of Science Education, 18(7), pp. 755-760.

HOWE, A.C. (1996). Development of science concepts within a Vigotskian framework. Science Education, 80(1), pp. $35-51$.

IZQUIERDO, M., SANMARTÍ, N. y ESPINET, M. (1999). Fundamentación y diseño de las prácticas escolares de ciencias experimentales. Enseñanza de las Ciencias, 17(1), pp. 45-90.

JIMÉNEZ, M.P. (1998). Diseño curricular: indagación y razonamiento en el lenguaje de las ciencias. Enseñanza de las Ciencias, 16(2), pp. 203-216.

JOUNG, W. (1993). Uses of cognitive science to science education. Science \& Education, 2(1), pp. 31-36.

KEMPA, R.F. y AYOB, A. (1995). Learning from group work in science. International Journal of Science Education, $10(3)$, pp. 275-284.

KLOPFER, L.E. (1983). Research and the crisis in science education. Science Education, 67(3), pp. 283-284.

LINN, M.C. (1987). Establishing a research base for science education: challenges, trends and recommendations. Journal of Research in Science Teaching, 24(3), pp. 191-216.

MARÍN, N. (1999). Delimitando el campo de aplicación del cambio conceptual. Enseñanza de las Ciencias, 17(1), pp. 80-92.

MARÍN, N. (1999b). Del cambio conceptual a la adquisición de conocimientos. Enseñanza de las Ciencias, 17(1), pp. 109-114.
MARTÍNEZ TERRADES, F. (1998). La didáctica de las ciencias como campo específico de conocimientos. Génesis, estado actual y perspectivas. Tesis doctoral. Universidad de Valencia.

MARTÍNEZ-TORREGROSA, J., DOMÉNECH, J.L. y VERDÚ, R. (1993). Del derribo de ideas al levantamiento de puentes: La epistemología de la ciencia como criterio organizador de la enseñanza de las ciencias. Qurriculum, 6 y 7, pp. 67-89.

MATTHEWS, M.R. (1994). Historia, filosofía y enseñanza de las ciencias: la aproximación actual.Enseñanzade las Ciencias, 12(2), pp. 255-277.

McDERMOTT, L.C., SHAFFER, P.S., ROSENQUIST, M.L. y PHYSICS EDUCATION GROUP(1996). Physics by inquiry. Nueva York: Wiley \& Sons, Inc.

MILLAR, R. (1989).Constructive criticism.International Journal of Science Education, 11, pp. 587-596.

MILLAR, R. y DRIVER, R. (1987). Beyond processes. Studies in Science Education, 14, pp. 33-62.

MORTIMER, E.F. (1995). Conceptual change or conceptual profile change? Science \& Education, 4(3), pp. 262-287.

NATIONAL RESEARCH COUNCIL (1996). National Science Education Standards. Washington, DC: National Academy Press.

NOLA, R. (1997). Constructivism in Science and Science Education: A Philosophical Critique. Science \& Education, 6, pp. 55-83.

NOVAK, J.D. (1988). Constructivismo humano: un consenso emergente. Enseñanza de las Ciencias, 6(3), pp. 213-223.

OLIVA, J.M. (1999a). Algunas reflexiones sobre las concepciones alternativas y el cambio conceptual. Enseñanza de las Ciencias, 17(1), pp. 93-107.

OLIVA, J.M. (1999b). Ideas para discusión sobre las concepciones de cambio conceptual. Enseñanza de las Ciencias, 17(1), pp. 115-117.

OSBORNE, J.F. (1996). Beyond constructivism. Science Education, 80(1), pp. 53-82.

OSBORNE, R. y WITTROCK, M. (1983). Learning Science: a generative process. Science Education, 67, pp. 490-508.

OSBORNE, R. y WITTROK, M. (1985). The generative learning model and its implications for science education. Studies in Science Education,12, pp. 59-87

PESSOA DE CARVALHO, A. y GIL-PÉREZ, D. (1995). Formaçâo de professores de ciências. Tendências e inovaçôes. São Paulo: Cortez Editora.

PIAGET, J. (1970). La epistemología genética. Barcelona: Redondo.

PIAGET, J. (1971). Psicología y epistemología. Barcelona: Ariel.

POPE, M.L. y GILBERT, J. (1983). Personal experience and the construction of knowledge in science. Science Education, 67, pp. 193-203.

PORLÁN, R. (1998). Pasado, presente y futuro de la didáctica de las ciencias. Enseñanza de las Ciencias, 16(1), pp. 175185 .

PORLÁN, R. (1993). Constructivismo y escuela. Hacia un modelo de enseñanza-aprendizaje basado en la investigación. Sevilla: Díada. 
POSNER, G.J., STRIKE, HEWSON y GERTZOG (1982). Accomodation of a scientific conception: towards a theory of conceptual change. Science Education, 66, pp. 211-227.

POZO, J.I. (1987). Aprendizaje de la ciencia y pensamiento causal. Madrid: Visor.

POZO, J,I. (1989). Teorías cognitivas del aprendizaje. Madrid: Morata.

POZO, J.I., GÓMEZ CRESPO, M.A., LIMÓN, M. y SERRANO SANZ, A. (1991). Procesos cognitivos en la comprensión de las ciencias: las ideas de los adolescentes sobre la química Madrid: CIDE.MEC.

RELA, A. y TRICÁRICO, H.R. (1997). Física I. Fuerza, movimiento y energía; Documentos para la Capacitación Docente. Buenos Aires: Universidad Nacional San Martín.

RESNICK, L.B. (1983). Mathematics and Science Learning: a new conception. Science, 220, pp. 477-478.

ROBINSON, W.R. y NIAZ, M. (1991). Performance based on instruction by lecture or by interaction and its relationship to cognitive variables. International Journal of Science Education, 13(2), pp. 203-215.

ROTH, W.M. Learning process studies: examples from Physics. International Journal of Science Education, pp. 1019-1024.

SALINAS, J., CUDMANI, L. y PESA, M. (1996). Modos espontáneos de razonar: un análisis de su incidencia sobre el aprendizaje del conocimiento físico a nivel universitario básico. Enseñanza de las Ciencias, 14(2), pp. 209-220.

SANMARTÍ, N. y AZCÁRATE, C. (1997). Reflexiones en torno a la línea editorial de la revista Enseñanza de las Ciencias. Enseñanza de las Ciencias, 15(1), pp. 3-9.

SHUELL, T.J. (1987). Cognitive Psychology and conceptual change: implications for teaching science. Science Education, 71(2), pp. 239-250.
SOLOMON, J. (1987). Social influences on the construction of pupils' understanding of science.Studies in Science Education, 14 , pp. 63-82.

SOLOMON, J. (1991). Teaching about the nature of science in the British National Curriculum. Science Education, 75(1), pp. 95-103.

SOLOMON, J. (1994). The rise and fall of constructivism. Studies in Science Education, 23, pp. 1-19.

SUCHTING, W.A. (1992). Constructivism deconstructed. Science \& Education, 1(3), pp. 223-254.

TIBERGHIEN, A., JOSSEM, E. y BAROJAS. J. (1998). Connecting Research in Physics Education with Teacher Education. ICPE Book. International Commission on Physics Education.

VIENNOT, L. (1989). L'enseignement des sciences physiques object de recherche. Bulletin de l'Union des Physiciens, 716, pp. 899-910.

VIENNOT, L. (1996). Raisonner en Physique. La part du sens commun. París y Bruselas: De Boeck \& Larcier, SA.

VIENNOT, L. (1999). Design and evaluation of a researchbased teaching sequence: the superposition of electric field.

WHEATLEY, G.H. (1991). Constructivist perspectives on Science and Mathematics learning. Science Education, 75(1), pp. 9-21.

WANDERSEE, J.H., MINTZES, J.J. y NOVAK, J.D. (1994). Research on alternative conceptions in science, en Gabel D.L. (ed.). Handbook of Research on Science Teaching and Learning. Nueva York: MacMillan Pub Co.

WHITE, R. (1999). The revolution in Research on Science teaching, en Richardson V. (ed.). Handbook of Research on Teaching (4a. ed., en preparación). 